\title{
A RECURSION FORMULA FOR FINITE PARTITION LATTICES
}

\author{
TERRENCE J. BROWN
}

A partition on a nonempty set $S$ is a collection of disjoint nonempty subsets, called blocks, whose union is $S$. The recursion formula

$$
B_{n}=\sum_{j=0}^{n-1}\left(\begin{array}{c}
n-1 \\
j
\end{array}\right) B_{j} \quad\left(n \geqq 1, B_{0}=1\right)
$$

for the number of partitions on a finite set with $n$ elements is well known (cf. Rota [4]). We obtain equation (2) which generalizes (1) and which yields a derivation of the Möbius function for partition lattices different from those in [2], [3] and [5].

Let $S$ be a finite nonempty set. Two partitions $\sigma$ and $\pi$ of $S$ satisfy $\sigma \leqq \pi$ if every block of $\sigma$ is contained in a block of $\pi$. $\leqq$ is a partial ordering of the collection $L(S)$ of partitions of $S$ and $(L(S), \leqq)$ is a lattice (cf. Birkhoff [1]). If $h$ is a function from the natural numbers into the integers $Z$, it is possible to define a function $k: L(S) \rightarrow Z$ by setting $k(\sigma)=\prod_{B \in \sigma} h(|B|)$ for $\sigma \in L(S) \quad(|B|=$ the cardinality of the block $B$ ). It is then possible to define another function $H$ from the nonnegative integers into $Z$ by setting $H(0)=1$ and $H(n)$ $=\sum_{\sigma \in L_{n}} k(\sigma)$ for $n \geqq 1$, where $L_{n}$ is the lattice of partitions on a set with $n$ elements.

THEOREM. If $h, k$ and $H$ are as above then

$$
H(n)=\sum_{j=0}^{n-1}\left(\begin{array}{c}
n-1 \\
j
\end{array}\right) h(j+1) H(n-1-j) .
$$

Proof. Assume now that finite nonempty $S$ has $n$ elements. First we show that if $C$ is a nonempty subset of $S$, then $\sum_{c \in \sigma \in L(S)} k(\sigma)$ $=h(|C|) H(|S-C|) . \quad C=S \quad$ implies $\quad \sum_{C \in \sigma \in L(S)} k(\sigma)=k\{S\}$ $=h(|S|) H(0)$. If $C \varsubsetneqq S$ then

$$
\begin{aligned}
\sum_{C \in \sigma \in L(S)} k(\sigma) & =\sum_{C \in \sigma \in L(S)} h(|C|) k(\sigma-\{C\}) \\
& =h(|C|) \sum_{\pi \in L(S-C)} k(\pi)=h(|C|) H(|S-C|) .
\end{aligned}
$$

Received by the editors September 25, 1968. 
Because $S$ is nonempty we may pick $a \in S$. Define $f: L(S) \rightarrow P(S-\{a\})$ (the power set of $S-\{a\}$ ) by $f(\sigma)=\sigma_{a}-\{a\}, \sigma \in L(S)$, where $\sigma_{a}$ is the block of $\sigma$ containing $a$. For

$$
D \in P(S-\{a\}) f^{-1}(D)=\{\sigma \in L(S) \mid D \cup\{a\} \in \sigma\} .
$$

As $f$ partitions $L(S)$,

$$
\begin{aligned}
H(n) & =\sum_{\sigma \in L(S)} k(\sigma)=\sum_{D \in P(S-\{a\})} \sum_{\sigma \in f^{-1}(D)} k(\sigma) \\
& =\sum_{j=0}^{n-1} \sum_{D \in P(S-\{a\}) ;|D|=j} \sum_{D \cup\{a\} \in \sigma \in L(S)} k(\sigma) \\
& =\sum_{j=0}^{n-1} \sum_{D \in P(S-\{a\}) ;|D|=j} h(|D \cup\{a\}|) . \\
H(\mid S-D & -\{a\} \mid)=\sum_{j=0}^{n-1}\left(\begin{array}{c}
n-1 \\
j
\end{array}\right) h(j+1) H(n-1-j) .
\end{aligned}
$$

Equation (2) now follows.

Putting $h(n)=1, n \geqq 1, k$ maps each partition $\sigma$ onto 1 and $H(n)$ becomes $B_{n}$ while equation (2) reduces to (1).

The 0 of $L_{n}$ is the partition having $n$ blocks while the 1 of $L_{n}$ is the partition having one block. See Rota [3] for the definition of the Möbius function $\mu$.

CoROllaRY. If $\mu_{n}=\mu(0,1)$ for the lattice $L_{n}$ of partitions on a set with $n$ elements, then $\mu_{n}=(-1)^{n-1}(n-1)$ !

Proof. Set $h(n)=\mu_{n}$ for $n \geqq 1$. $\sigma \in L_{n}$ implies that

$$
k(\sigma)=\prod_{B \in \sigma} h(|B|)=\prod_{B \in \sigma} \mu_{|B|}=\mu(0, \sigma)
$$

(cf. Rota [3], especially Proposition 5, p. 345, and the lemma on p. 359). Since $H(m)=\sum_{\sigma \in L m} k(\sigma)=\sum_{g \in L m} \mu(0, \sigma)=0$ for $m \geqq 2$, $H(0)=1$ and $H(1)=\mu_{1}=1$, we see from (2) that

$$
0=H(n)=\left(\frac{n-1}{n-2}\right) h(n-1) H(1)+\left(\begin{array}{l}
n-1 \\
n-1
\end{array}\right) h(n) H(0),
$$

i.e., that $\mu_{n}=-(n-1) \mu_{n-1}$ for $n \geqq 2$. From $\mu_{1}=1$ the corollary follows. 


\section{REFERENCES}

1. Garrett Birkhoff, Lattice theory, rev. ed., Amer. Math. Soc., Providence, R. I., 1966.

2. Roberto Frucht and Gian-Carlo Rota, La función de Möbius para el retículo di particiones de un conjuncto finito, Scientia; revista cientifica y técnica (Chile) N. 122 (1963), 111-115.

3. Gian-Carlo Rota, On the foundations of combinatorial theory. I, Theory of Möbius functions, Z. Wahrscheinlichkeitstheorie 2 (1964), 340-368.

4. - The number of partitions of a set, Amer. Math. Monthly 71 (1964), 498504.

5. M. P. Schutzenberger, Contribution aux applications statistiques de la theorie de l'information, Publ. Inst. Statist. Univ. Paris 3 (1954), 5-117.

University of Missouri at Kansas City 